OPEN ACCESS

Edited by:

Eric Ruelland,

Centre National de la Recherche

Scientifique (CNRS), France

Reviewed by:

Kazuo Nakashima,

Japan International Research Center

for Agricultural Sciences, Japan

Hikmet Budak,

Montana State University,

United States

${ }^{*}$ Correspondence:

Huaijun Si

hjsi@gsau.edu.cn

tThese authors have contributed equally to this study.

Specialty section: This article was submitted to

Plant Abiotic Stress,

a section of the journa

Frontiers in Plant Science

Received: 11 January 2018 Accepted: 08 March 2018

Published: 29 March 2018

Citation:

Zhang L, Yao L, Zhang N, Yang J,

Zhu X, Tang X, Calderón-Urrea A

and Si $H$ (2018) Lateral Root

Development in Potato Is Mediated

by Stu-mi164 Regulation of NAC

Transcription Factor.

Front. Plant Sci. 9:383.

doi: 10.3389/fpls.2018.00383

\section{Lateral Root Development in Potato Is Mediated by Stu-mi164 Regulation of NAC Transcription Factor}

\author{
Li Zhang 1,2t, Lei Yao 1,2t, Ning Zhang 1,2, Jiangwei Yang 1,2, Xi Zhu'1,2, Xun Tang 1,2, \\ Alejandro Calderón-Urrea ${ }^{3,4}$ and Huaijun $\mathrm{Si}^{1,2 *}$
}

1 Gansu Provincial Key Laboratory of Aridland Crop Science, Gansu Key Laboratory of Crop Genetic and Germplasm Enhancement, Gansu Agricultural University, Lanzhou, China, ${ }^{2}$ College of Life Science and Technology, Gansu Agricultural University, Lanzhou, China, ${ }^{3}$ College of Plant Protection, Gansu Agricultural University, Lanzhou, China, ${ }^{4}$ Department of Biology, California State University, Fresno, CA, United States

The NAC designation is derived from petunia (Petunia hybrida) gene NO APICAL MERISTEM (NAM) and Arabidopsis genes ATAF1/ATAF2 and CUP-SHAPED COTYLEDON2 (CUC2), which belongs to the family of plant-specific transcription factors (TFs), and plays important role in plant development processes, such as response to biotic and abiotic stress, and hormone signaling. MicroRNAs (miRNAs) are a class of small, non-coding endogenous RNAs which play versatile and significant role in plant stress response and development via negatively affecting gene expression at a post-transcriptional level. Here, we showed that Stu-mi164 had a complementary sequence in the CDS sequence of potato NAC TFs, and that NAC expression exhibited significant differences under osmotic stress. We measured expression levels of the Stu-mi164 target gene StNAC262 between control and PEG-treated plants using real-time PCR, and the results demonstrated that they had inverse relationship. We suggested that Stu-miR164 might drive overexpression of NAC gene under osmotic stress in potato. To confirm the regulation of NAC TFs by Stu-mi164, we developed transgenic plants, using Agrobacterium tumefaciens-mediated transformation, of the potato cultivars "Gannongshu 2" and "Kexin 3" overexpressing the Stu-mi164 or the TF StNAC262. Real-time PCR analysis of transgenic potato plants under osmotic (PEG) stress, showed that potato plants overexpressing Stu-mi164 had reduced expression of StNAC262 and their osmotic resistance decreased. Furthermore, these plants had low number of lateral roots although the same length as the control. Our findings support the regulatory role of Stu-miRNAs in controlling plant response to osmotic stress via StNAC262.

Keywords: miRNA, NAC, osmotic stress, lateral root, potato

\section{INTRODUCTION}

Drought stress is one of the main factors severely affecting plant physiology and biochemistry, ultimately limiting plant growth and development and causing decrease in crop production efficiency (Thao and Tran, 2012). Plants have developed sophisticated mechanisms to deal with diverse unfavorable environmental factors. A number of physiological and 
metabolic processes of plant were triggered to promote plant survival under water deficit (Shinozaki and Yamaguchi, 2007). Numerous genes of plant, including transcription factor (TF) genes, have altered their expression for stress adaptation (Yang et al., 2010). TFs play vital role in stress responses of plant by binding to the cis-acting element located in promoter region of downstream target genes to regulate various signaling pathways, thereby activating them, and by interacting with other proteins (Yamaguchi-Shinozaki and Shinozaki, 2006).

About 20 years ago, the specific NAC TF family of plant was first described in Petunia (Souer et al., 1996). Many studies have found that most of the NAC TFs genes are influenced by a variety of biological and abiotic stress which shows that they are plays important roles in stress response and signal transduction (Tran et al., 2004; Liu et al., 2011). We deleted the reference Chatterjee et al., 2011. The genomic sequencing results allowed identifying NAC family members in a number of sequenced species, for example, 163 genes in poplar (Populus trichocarpa) (Hu et al., 2010), 152 genes in tobacco (Nicotiana tabacum) (Rushton et al., 2008), 151 genes in rice (Oryza sativa) (Nuruzzaman et al., 2010), 117 genes in Arabidopsis and about 200 genes in soybean (Glycine max) (Mochida et al., 2009). The NAC TFs are multi-functional proteins which are involved in response to diverse cues, including biotic and abiotic stress responses (Olsen et al., 2005), lateral root formation and auxin signaling (Xie et al., 2000), secondary cell wall synthesis regulation, cell division (Zhong et al., 2007), embryo development (Duval et al., 2002), and flowering (Yoo et al., 2007). The NAC TFs have a conserved DNA-binding domain and a variable domain at the end $\mathrm{N}$-terminal and $\mathrm{C}$-terminal end, respectively, which are important for the transcriptional regulatory roles (Duval et al., 2002).

Xie et al. (2000) found that NAC1 gene in Arabidopsis thaliana was induced by auxin and mediated auxin signaling in order to promote development of lateral root. This TF can activate two downstream auxin response genes $D B P$ (DNA binding protein) and AIR3 (auxin-induced in root cultures 3 ) to express. Excessive expression of NAC1 can promote lateral root development, while antisense expression inhibits lateral root development. $D B P$ encodes a DNA binding protein and AIR3 encoding a class of hay bacillus protease that can weaken the connections between cells, indirectly promoting the development of lateral roots (Alliotte et al., 1989). In addition, Arabidopsis thaliana AtNAC2 gene is specifically expressed in the root after induction by ethylene signaling and auxin signaling pathways, which can significantly increase the number of lateral root (He et al., 2005). Moreover, NAC TF was induced by a variety of biotic stress, involved in plant stress response. Hu et al. (2006) cloned a rice drought resistance and salt tolerance gene $S N A C 1$, which is a type of NAC TF expressed primarily in stomatal guard cells; it was proposed drought stress promote stomatal closure but does not affect the photosynthetic rate, thus greatly improving drought resistance. Using yeast single hybrid technology, Tran et al. (2004) isolated three different NAC genes from Arabidopsis thaliana (ANAC019, ANAC055, and ANAC072). Overexpression of three NAC TFS gene in Arabidopsis thaliana can enhance the drought-resistant ability of genetically modified plants. High expression levels of these genes in the transgenic plants induced by drought, high salt, and ABA, significantly enhanced the drought-resistant ability of the plants (Tran et al., 2004).

MicroRNAs (miRNAs) are small and endogenous RNAs, which play vital regulatory function in stress responses of plant through negatively affecting gene expression at the level of post-transcription (Voinnet, 2009), by degrading transcript of the target genes (Llave et al., 2002), and therefore play an important role in attenuating translation (Chen, 2004). miRNAs can bind to their target transcripts by complementary base pairing, and either direct the target cleavage or repress its translation, further leading to the decreased expression of the target gene; therefore, miRNAs can act at the levels of both transcription and post-transcription (Hikmet et al., 2015). There are few agronomic traits in crops are controlled by single gene or isolated biological pathway. Cellular responses to stresses usually involve complex networks of gene interaction which are regulated at multiple levels (Budak et al., 2015). In other plant species, conservation of miRNAs in sequence and structure provides strong characteristics for the prediction of new miRNAs (Kantar, 2010). To date, there are thousands of miRNAs and the majority of their target genes that have been identified. Many miRNA target transcripts have been associated with stress-responsive TF families including WRKY and NAC in Triticeae (Kantar et al., 2011; Deng et al., 2015). The target genes can encode important enzymes or TFs, which play important roles in plant development, including floral, stem, leaf and root development (Chen, 2004), signal transduction (Rhoades et al., 2002), auxin (Guo et al., 2005) and disease responses (Chapman et al., 2004), or various abiotic and biotic stresses (Jones-rhoades and Bartel, 2004). Most researches have been focused on model plants, such as rice (Oryza sativa) and Arabidopsis thaliana (Bartel and Bartel, 2003; Bonnet et al., 2004; Griffiths et al., 2006). Many conserved miRNAs of plant, such as miR156, miR159, or miR164, have been shown to target stress-related TFs including MYB and NAC family members (Burcu et al., 2016). In potato crop, few miRNAs have been found in drought stress or other abiotic stress responses (Hwang et al., 2011; Zhang et al., 2014). Moreover, there are no known miRNAs regulating NAC TFs under osmotic stress in potato.

We are interested in identifying novel miRNA genes that can regulate stress response and try to generate stress-resistant or tolerant potatoes by transgenic approach. In Brassica and rice seedlings, miR164 was shown to target a NAC TF, whose expression was negatively correlated with miR164 under stress conditions including drought, salinity, and high-temperature (Bhardwaj et al., 2014; Fang et al., 2014). In the present study, based on a miRNA bioinformatics analysis, we identified possible miRNAs that can regulate the NAC gene. We discovered that the potato Stu-mi164 had significant differences in expression levels under osmotic (PEG) stress. Target prediction also identified that it has a binding site on the CDS sequence of the NAC gene in potato. We further detected the expressional levels of the NAC gene response under osmotic (PEG) stress by qRT-PCR. We concluded that decreased expression of Stu-miR164 drives overexpression of the potato $N A C$ gene and can help plant response to osmotic stress. 


\section{MATERIALS AND METHODS}

\section{Plant Materials and Growth Conditions}

The potato cultivars "Gannongshu 2" with more lateral roots and "Kexin 3" with fewer lateral roots were used in the experiment. The potato plantlets were propagated by sub-culturing using single-node cuttings on MS media containing 3\% sucrose and $0.45 \%$ agar. Plantlets were grown in $150 \mathrm{~mL}$ flasks under $16 / 8 \mathrm{~h}$ light/dark cycle of white fluorescent lamp at $(25 \pm 2)^{\circ} \mathrm{C}$. Microtubers were induced on MS media containing $8 \%$ sucrose and $0.45 \%$ agar under dark conditions at $(25 \pm 2)^{\circ} \mathrm{C}$ (Zhang et al., 2005).

\section{Bioinformatics and Software}

The miRNA sequences of potato were obtained from the miRNA registry miRBase ${ }^{1}$ (Saini et al., 2008). The target genes of Stu-mil64 were predicted using online program psRNATarget ${ }^{2}$ (Dai and Zhao, 2011). WMD3 ${ }^{3}$ (Web app for the automated design of artificial microRNAs) was use to design Stu-mil64 cloning primers. Sequence of potato and Arabidopsis NAC family TFs were obtained from plant TF database ${ }^{4}$ (Jin et al., 2014). The analysis of chromosome structure uses PGSC 5 . Alignment analysis and phylogenetic tree construction were achieved using CluxtalX 1.8 and MEGA 5.0 program. The data of qRT-PCR was analyzed by statistics software SPSS version 13.0.

\section{Subcellular Localization Assay}

The coding sequence of StNAC262 was amplified using genespecific primers NAC262-F and NAC262-R and inserted into vector pEGFP at the $B a m \mathrm{H}$ I and $\mathrm{Xba}$ I sites, yielding plasmid pEGFP-NAC262. The plasmid pEGFP-NAC262 and pEGFP were transformed into Agrobacterium tumefaciens LBA4404 and the agrobacteria were infiltrated, separately, into tobacco (Nicotiana benthamiana L.) leaves using 1-mL needless syringes. After agroinfiltration, the plants were grown under room temperature $\left(25^{\circ} \mathrm{C}\right)$ for $48 \mathrm{~h}$ in darkness. GFP fluorescence signals were excited at $488 \mathrm{~nm}$ and detected under a Leica SP8 confocal laser scanning microscope (Germany).

\section{Construction of the Plant Expression Vectors}

The potato StNAC262 gene sequence (GenBank accession number XM_006364522.1) was obtained from $\mathrm{NCBI}^{6}$ nucleic acid database. PCR primers were designed based on the StNAC262 gene sequence using software Primer 5.0. The enzyme sites of StNAC262 gene sequence were analyzed based the expression vectors $\mathrm{pCPB} 121$ and $\mathrm{pCPB}$ sequences to add the appropriate restriction sites $\mathrm{Xba} \mathrm{I}, \mathrm{BamH} \mathrm{I}$, and protection bases at both ends of the primers. The PCR primers sequences as follows: StNAC262-F: TCTAGACCATCTCCTTCAAAGACCAC

${ }^{1}$ http://microrna.sanger.ac.uk/

${ }^{2}$ http://plantgrn.noble.org/psRNATarget/

${ }^{3}$ http://wmd3.weigelworld.org/cgi-bin/webapp.cgi

${ }^{4}$ http://planttfdb.cbi.pku.edu.cn/

${ }^{5}$ http://solanaceae.plantbiology.msu.edu/pgsc_download.shtml

${ }^{6}$ http://www.ncbi.nlm.nih.gov and StNAC262-R: GGATCCGCAGTGAGACAAATGGGAA (the underlined section showed enzyme sites). The Stu-mi164 primers was design on website named WMD3, and primer A (CTGCAAGGCGATTAAGTTGGGTAAC), B (GCGGATAACA ATTTCACACAGG AAACAG), I (GATGGAGAAGCAGGGC ACATGCTTCTCTCTTTTGTATTCC), II (GAAGCATGTGC CCTGCTTCTCCATCAAAGAGAATCAATGA), III (GAAGA ATGTGCCCTGGTTCTCCTTCACAGGTCGTGATATG), and IV (GAAGGAGAA CCAGGGCACATTCTTCTACATATATATT CCT) (the underlined section showed the sequence of Stu-mi164) were used for overlapping PCR according to the method provided on the website to construct the Stu-mil64 cloning vector. The primers were synthesized by the Shanghai Biological Engineering Co., Ltd. The re-modified StNAC262 gene and Stu-mi164 was driven by the constitutive CaMV $35 \mathrm{~S}$ promoter. The engineered vectors, named pCPB-NAC262 and pCPB121-miR164, were, respectively, transformed into Escherichia coli $\mathrm{DH} 5 \alpha$, which was identified by double enzyme digested for further using. After identification, both of the two vectors were transformed into Agrobacterium tumefaciens LBA4404 by the freeze-thaw method (Wang et al., 2011).

\section{Potato Transformation and Identification of the Transgenic Plants}

Potato transformation was performed using Agrobacteriummediated method (Si et al., 2003). The microtubers of potato cultivars "Gannongshu 2" and "Kexin 3" were peeled and removed buds part, then cut into slices with thickness of 1-2 mm. The slices soaked into the Agrobacterium containing the vectors pCPB121-miR164 and pCPB-NAC262, after $7 \mathrm{~min}$ infection, the tissue slices were dried with filter paper placed into MS solid media at $28^{\circ} \mathrm{C}$ in the dark for $48 \mathrm{~h}$. After co-cultivation the tissue slices were placed in differentiation media (MS + $1 \mathrm{mg} / \mathrm{L} \mathrm{IAA}+0.2 \mathrm{mg} / \mathrm{L} \mathrm{GA}_{3}+0.5 \mathrm{mg} / \mathrm{L} 6-$ $\mathrm{BA}+2 \mathrm{mg} / \mathrm{L} \mathrm{ZT}+50 \mathrm{mg} / \mathrm{L} \mathrm{Kan}+500 \mathrm{mg} / \mathrm{L}$ carbenicillin), at $25^{\circ} \mathrm{C}$ for $2500 \mathrm{~lx}$ light to culture, and transferred every weeks to replace media. When the new buds were derived from the center of potato slice (Supplementary Figure S1a), they were transferred into rooting media containing $75 \mathrm{mg} / \mathrm{L}$ kanamycin and $200 \mathrm{mg} / \mathrm{L}$ carbenicillin for rooting screen about 7 days (Supplementary Figure S1b) when the buds were transferred to a selective rooting media.

DNA extraction from the transgenic plants was performed using the CTAB method. The total DNA of transformed potato as a template and untransformed as a negative control. The resistant plants obtained by neomycin phosphate transferase (NPT II) gene of a pair of primers (NPT II-F: GCTATGACTGGGCACAACAG) and (NPT II-R: ATACCGTAAAGCACGAGGAA) using for PCR to detections and the expected fragment size was $676 \mathrm{bp}$. The PCR reaction conditions: incubated at $94^{\circ} \mathrm{C}$ for $3 \mathrm{~min}$, followed by 35 cycles of $94^{\circ} \mathrm{C}$ for $45 \mathrm{~s}, 60^{\circ} \mathrm{C}$ for $45 \mathrm{~s}$, and $72^{\circ} \mathrm{C}$ for $1 \mathrm{~min}$, then $10 \mathrm{~min}$ final extension at $72^{\circ} \mathrm{C}$ detecting by $1 \%$ agarose gel electrophoresis. We choose the rooting potatoes for further study and named as T1/T2 from "Gannongshu 2" transformed using the vector pCPB121-miR164, and T3/T4 from "Kexin 3" transformed using the vectors pCPBNAC262. 


\section{Transgenic Potato Growth and Osmotic (PEG) Stress Treatment}

Transgenic potatoes were propagated on liquid MS media supplemented with $3 \%$ sucrose without kanamycin. Plantlets were fixed with filter paper and grown in $150 \mathrm{ml}$ flasks under a $16 / 8 \mathrm{~h} \mathrm{light/dark}$ cycle of white fluorescent lamp at $(25 \pm 2)^{\circ} \mathrm{C}$. After 3 weeks, the liquid media were poured out, and $50 \mathrm{ml}$ of fresh MS liquid media supplemented with 3\% sucrose and 20\% PEG6000 (polyethylene glycol) was added. Plantlets were cultured under the same conditions for $0,4,8,16$, and $32 \mathrm{~h}$. The roots, leafs, and stems were collected and frozen in liquid nitrogen for preservation to extract RNA.

\section{qRT-PCR Analysis of the Transgenic Potato Plants}

Expression of NAC family members was assayed using qRT-PCR with SuperReal PreMix Plus (SYBER Green) (TIANGEN). Briefly, $20 \mu \mathrm{l}$ PCR contained about $100 \mathrm{ng}$ cDNA, $10 \mu \mathrm{l} 2 \times$ SuperReal PreMix Plus, $0.6 \mu \mathrm{l}$ each primer (NAC262-F: AGGGCTTGTGAGAGGGAATT, NAC 262-R: CGTGACTTGGCGATGAATC, efla-F: CAAGGATGA CCCAGCCAAG, efla-R: TTCCTTACCTGAACGC CTGT), and $0.4 \mu \mathrm{l} 50 \times \mathrm{RO} \times$ Reference Dye. The reactions were mixed gently and incubated at $95^{\circ} \mathrm{C}$ for $5 \mathrm{~min}$, followed by 40 cycles of $95^{\circ} \mathrm{C}$ for $30 \mathrm{~s}, 60^{\circ} \mathrm{C}$ for $34 \mathrm{~s}$, and $72^{\circ} \mathrm{C}$ for $30 \mathrm{~s}$. ef $1 \mathrm{a}$ gene was used as an reference gene as control for each sample. The reaction was performed using the Mx3005p Real-Time PCR System. The relative expression level of target genes was determined using the $2^{-\Delta \Delta C_{\mathrm{t}}}$ method. All samples were performed in three biological replicates with three technical replicates. The standard deviations of the data were obtained from three independent experiments. Statistical significance of individual gene expression differences upon osmotic stress was analyzed with one-way analysis of variance (ANOVA) using statistics software SPSS version 13.0.

\section{Physiological Assay of the Transgenic Potato Plants}

The transgenic and non-transgenic potato plants were propagated by sub-culturing using single-node cuttings on MS media supplemented with $3 \%$ sucrose. Plants were grown in $150 \mathrm{ml}$ flasks under $(25 \pm 2)^{\circ} \mathrm{C}$ and $2500 \mathrm{~lx}$ light. After 3 weeks growth, the lateral root number and length was determined. The experiments had five biological replicates. Significant differences were assayed by the Duncan's Multiple Range Test at the 5\% probability level.

\section{RESULTS}

\section{The Prediction of Stu-mi164 Target Genes}

Most miRNAs are known as negative regulators of genes. miR161, miR168, miR169, miR171a, and miR319c of Arabidopsis thaliana are downregulated under drought stress (Sunkar and Zhu, 2004; Liu et al., 2008), and in rice and maize, miR156 is downregulated under drought stress (Wei et al., 2009; Zhou et al., 2010). The short sequence length of these miRNAs makes it relatively easy for them to combine base pairs of other sequences, potentially allowing multiple gene regulation (Ferdous et al., 2015). We reason that similar miRNAs may be present in potato with the potential to regulate the response to drought stress. The potato Stu-mil64 sequence was obtained from the miRNA registry, miRBase, and the secondary hairpin structures of Stu-mi164 is shown in Supplementary Figure S2. We used the online program psRNATarget to identify three predicted target genes StNAC262 (XM_006364522.1), StNAC083 (XM_00635471 0.1), and StNAC280 (NM_001288405.1) from Solanum tuberosum (Supplementary Table S1). Since there are three possible targets, it is difficult to ascertain the biological role of the miRNA, and therefore, we adopted a transgenic approach to determine the role of Stu-mi164 and its three putative target genes.

\section{Phylogenetic Analysis of Stu-mi164 Target Gene NAC TFs}

In order to know more about the evolutionary relationship of the three NAC TFs, which we predicted as the target genes of Stu-mi164, all the StNAC and AtNAC TFs were subjected to multiple sequence alignments using the CluxtalX 1.8 and MEGA 5.0 program. The multiple sequence alignment file was used to construct unrooted phylogenetic tree by the neighbor-joining method. As shown in Supplementary Figure S3, we found that the three target genes all belong to the NAM subfamily of potato NAC TFs. StNAC262 and StNAC083 are highly conserved and share a highly similar gene structure, while the StNAC262 gene structure seemed to have experienced changes during evolution, but its coding protein still has the characteristics of NAC TFs.

\section{Gene Structure Analysis of StNACs}

The analysis of chromosome structure showed that StNAC083 was located on the 6th chromosome, StNAC262 on the 3rd chromosome, and StNAC280 on the 7th chromosome. The result of comparison between the genomic and cDNA sequences indicated that StNAC262 and StNAC083 had three exons, while StNAC280 had two exons (Figure 1). StNAC262 had one 920 bp length first exon, while StNAC083 had one 967 bp first exon. The two StNAC genes with three exons had a 278 bp highly conserved second exon, while StNAC280 did not have this exon. The predicted secondary structure of the corresponding proteins suggested that the proteins derived from StNAC262 and StNAC083 share a high degree of structural similarity (Table 1). Multiple sequence alignment with the StNAC262 protein shows high homology with Solanum lycopersicum SlNAC100 (96\%), Nicotiana sylvestris NsNAC100-like (84\%), and Nicotiana tomentosiformis NtNAC100-like (82\%) (Supplementary Figure S4).

\section{Nucleus Localized of NAC262-EGFP Fusion Protein}

To examine the subcellular localization of StNAC genes, we developed NAC-EGFP fusion proteins of StNAC262, StNAC083, and StNAC280. Agrobacterium tumefaciens carrying the StNAC262-EGFP fusions (or EGFP as a negative control) 


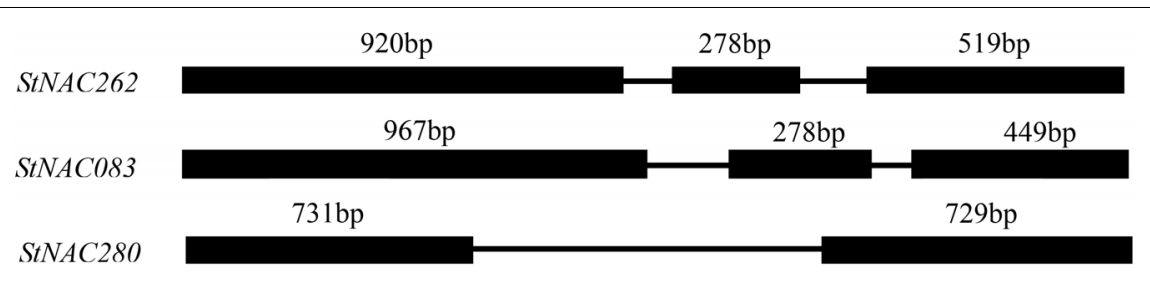

FIGURE 1 | Structures of StNACs genes in potato. Introns and exons were represented by lines and black boxes, respectively. The numbers above the exons indicate the length (bp) of the exons.

TABLE 1 | Basic properties and characteristics of the three StNACs genes in potato.

\begin{tabular}{|c|c|c|c|c|c|c|c|c|c|}
\hline Gene & Chr & NCBI GI & Genomic locus & Cds (bp) & Exons (No) & Full length (bp) & Amino acid (aa) & pl & Mw (Da) \\
\hline StNAC262 & 3 & 565398031 & PGSC0003DMT400050262 & 1002 & 3 & 1714 & 333 & 8.31 & 38337.5 \\
\hline StNAC083 & 6 & 565376561 & PGSC0003DMT400083083 & 1011 & 3 & 1694 & 336 & 8.13 & 37842.3 \\
\hline StNAC280 & 7 & 568215643 & PGSC0003DMT400032280 & 1062 & 2 & 1523 & 353 & 8.34 & 39761.6 \\
\hline
\end{tabular}

plasmid were infiltrated into 4 -week-old Nicotiana benthamiana plant leaves. The result of confocal micrographs showed that the EGFP-NAC262 fusion protein was solely and clearly localized to the nucleus, and the GFP alone distributed ubiquitously throughout the cell, and no specific compartmental localization (Figure 2). However, the StNAC083 and StNAC280 gene fusions did not exhibit any recognizable localization pattern; it is possible that these two sequences were pseudogenes with no function. The data demonstrated that the StNAC262 protein was localized to nucleus of the tobacco cells.

\section{Expression of StNAC262 Is Controlled by Stu-mi164 and It Is Overexpressed Under PEG Stress}

We first wanted to determine whether overexpression of Stu-mi164 would affect StNAC262 expression. The transgenic potato plants (T1 and T2 overexpressing miR164, and T3 and T4 overexpressing StNAC262) and the non-transgenic potato plants were propagated in vitro by sub-culturing single-node cuttings on MS media containing 3\% sucrose and cultured for 3 weeks. qRT-PCR analysis demonstrated that the expression of StNAC262 gene in the transgenic potato lines $\mathrm{T} 1$ and $\mathrm{T} 2$, which overexpress miR164, was downregulated (0.58- to 0.25 -fold) compared with the control, particularly in the roots of T1 (0.25-fold) (Figure 3 ). On the other hand, the expression of StNAC262 in T3 and T4, which overexpress StNAC26, was upregulated (1.97- to 3.53-fold); the roots of T4 plants were upregulated 3.53-fold. This data indicated to us that indeed Stu-mil64 regulates expression of StNAC262 (Figure 3).

We then wanted to test whether StNAC262 was overexpressed under PEG stress, similarly to the overexpression of the three NAC TFs genes in Arabidopsis thaliana (Tran et al., 2004). We selected five stress-treatment durations and compared their expression in the StNAC262 transgenic and WT plants under 20\% PEG6000 conditions. As shown in Figure 4, after $8 \mathrm{~h}$ under stress they reached the highest level of expression, in addition, the expression in roots was higher than leaf and stem. Furthermore, expression of StNAC262 in transgenic miR164

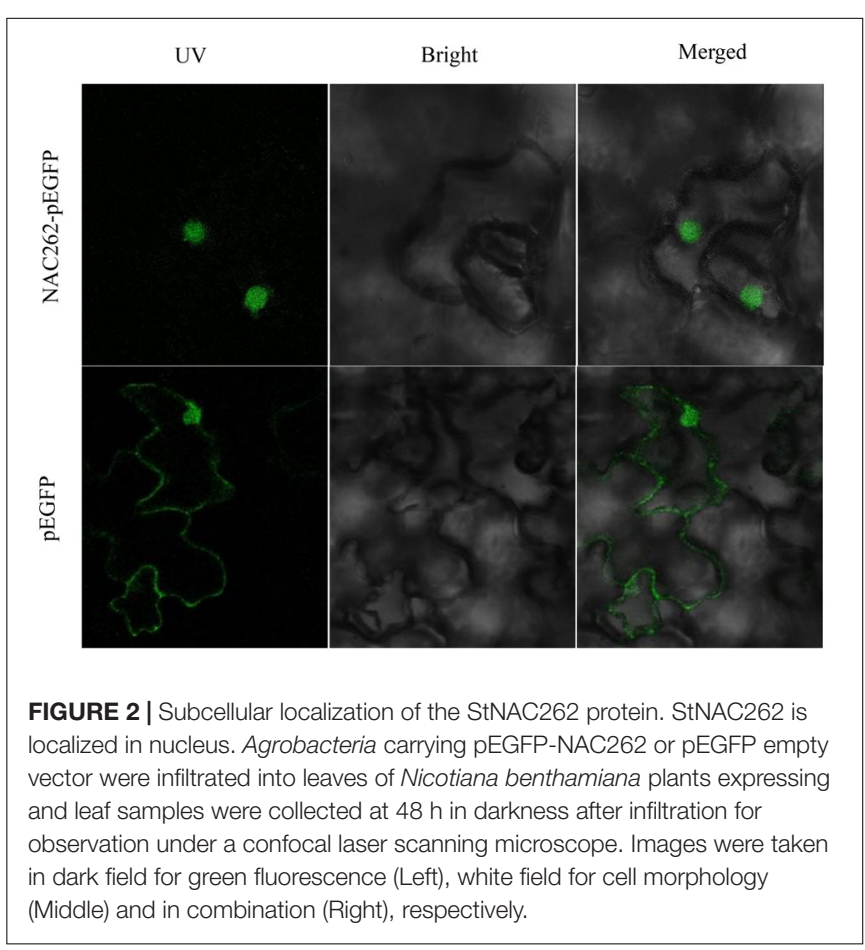

plants was downregulated under PEG stress (Figure 4A), while the drought stress increased significantly the expression of StNAC262 in transgenic StNAC262 plants compared with those in WT plants (Figure 4B). Taken together, the data indicated that Stu-mi164 was negative regulator of gene expression in transgenic potato and that overexpression of Stu-mi164 in potato suppresses the expression of StNAC2 and thus it conferred resistance to osmotic stress.

\section{Changes in Lateral Roots Associated With the Transgenic Potato Plants}

We successfully generated transgenic "Kexin 3" plants with the vector pCPB-NAC262, and transgenic “Gannongshu 2" plants 


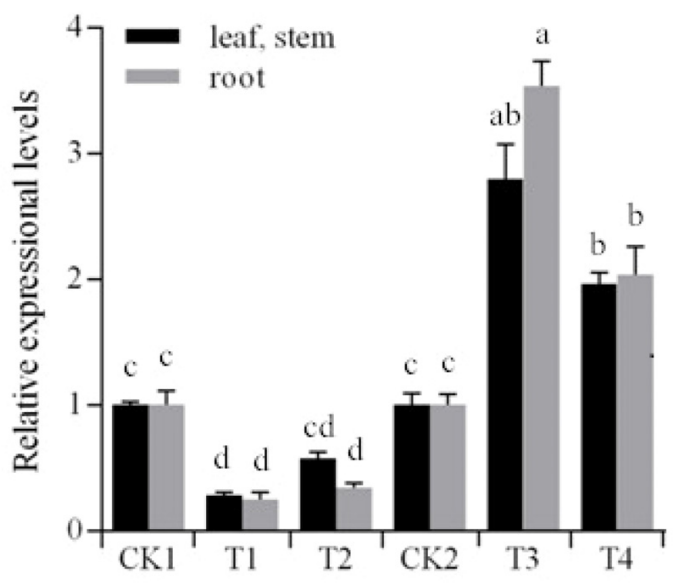

FIGURE 3 | Expressional levels of StNAC262 gene between the transgenic and non-transgenic potato plants. CK1: non-transgenic potato "Gannongshu 2" as negative control; T1-T2: the transgenic potato plant transformed with the vector pCPB121-miR164; CK2: non-transgenic potato "Kexin 3" as negative control; T3-T4: the transgenic potato plant transformed with the vector pCPB-NAC262. Data presented as a mean \pm SD (three biological replicates), different letters above the columns indicate significant differences at $p \leq 0.05$ levels with corresponding $\mathrm{CK}$.

with the vector pCPB121-miR164. These transgenic potato plants, which were propagated on MS media supplemented with $3 \%$ sucrose, had significant changes with respect to the lateral roots, not only in length but also in number (Figures 5, 6). The overexpressions of StNAC262 in "Kexin 3" result in a high number of lateral roots although shorter than the control, while overexpressions of miR164 in "Gannongshu 2" result in a low number of lateral roots although the same length as the control (Figure 4). These results are remarkable considering the fact that the potato cultivar "Gannongshu 2" naturally develops more lateral roots while "Kexin 3" naturally develops fewer lateral roots.

\section{DISCUSSION}

The level and activity of NAC TFs must be properly controlled for normal plant development and growth, and the mechanisms regulating NAC activity are becoming apparent (Laufs et al., 2004; Peaucelle et al., 2007; Larue et al., 2009). In the case of potato, predicted target genes StNAC262, StNAC083, and StNAC280 and their coding proteins have the characteristics of NAC TFs. In this study, we showed that only one of the genes shows subcellular nuclear localization in potato; we have no indication that the other two genes are functional and could be pseudogenes. Knowledge of NAC TFs target genes is still limited; however, it was reported that NAC1 regulated the expression of two auxinresponsive genes $D B P$ and $A I R 3$ (Xie et al., 2000). In the case of potato, it is likely that the genes involved in lateral root development are targets of StNAC262.

The same miRNA in the same plant species under osmotic stress is differentially expressed which may from the result
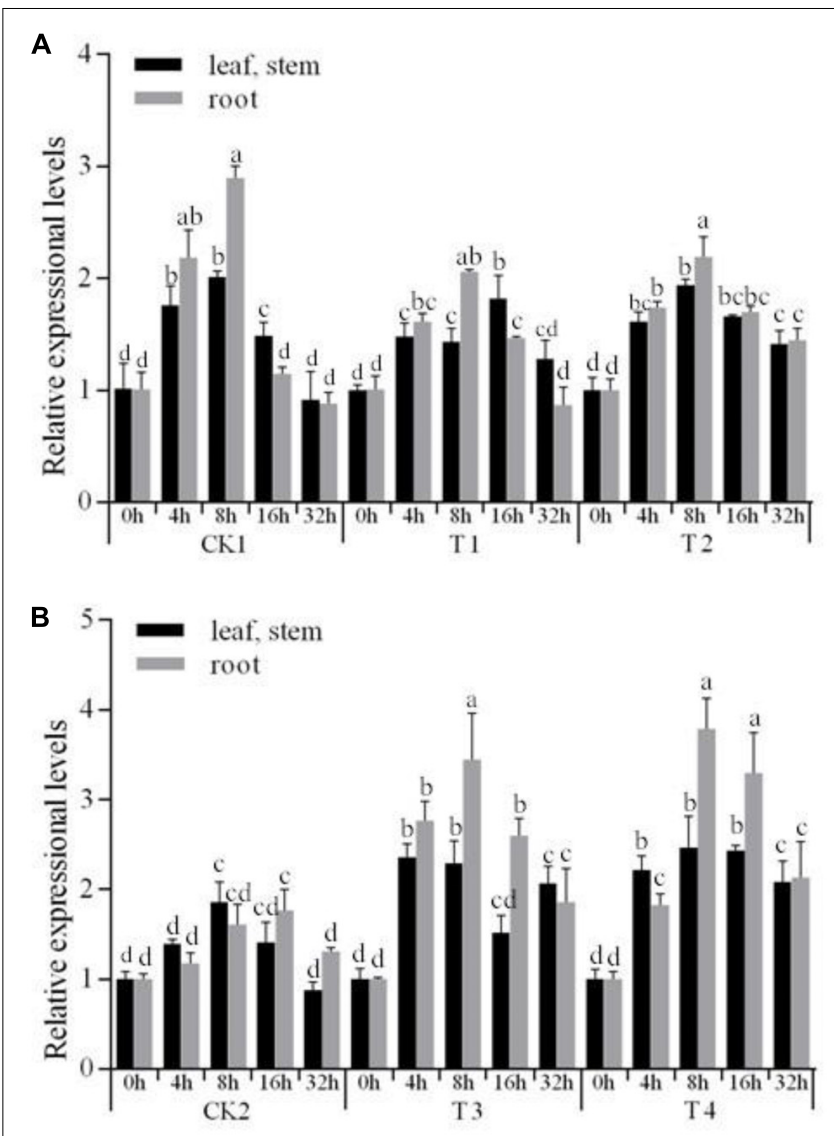

FIGURE 4 | Expression levels of StNAC262 gene under 20\% PEG6000 stress treatment. Relative expressional levels of StNAC262 in response to osmotic stress treatment for $0,4,8,16$, and $32 \mathrm{~h}$. (A) qRT-PCR analysis of potato "Gannongshu 2." CK1: non-transgenic potato "Gannongshu 2" as negative control; T1-T2: the transgenic potato plant transformed with the vector pCPB121-miR164. (B) qRT-PCR analysis of potato "Kexin 3." CK2: non-transgenic potato "Kexin 3" as negative control; T3-T4: the transgenic potato plant transformed with the vector pCPB-NAC262. Data presented as a mean \pm SD (three biological replicates), different letters above the columns indicate significant differences at $p \leq 0.05$ levels with corresponding CK.

of different spatial-temporal manner. The target genes of Arabidopsis thaliana miR164 family are five genes which coding NAM/ATAF/CUC (NAC) structure domain protein mRNA (Laufs et al., 2004). Many conserved miRNAs, such as miR156, miR159, or miR164, have proved to target stress-associated MYB and NAC family members TFs across monocots and dicots (Burcu et al., 2016). Plants excessive expressed miR164a and miR164b cotyledon and floral organ appear the phenomenon of fusion phenotypes which is similar to the CUC1 and CUC2 double mutant phenotypes, at the same time CUC1 and CUC2 transcript is decreased obviously (Ohnishi et al., 2005). Arabidopsis thaliana miR164 plays an important role in the process in regulating the meristem border size, differentiation of embryo and formation of floral organ (Ohnishi et al., 2005). miR164 identified from drought-stress roots of wheat can be used as candidate to develop tolerant variety to explore and exploit the drought response for future studies (Akpinar et al., 2015). The mutant of Arabidopsis 

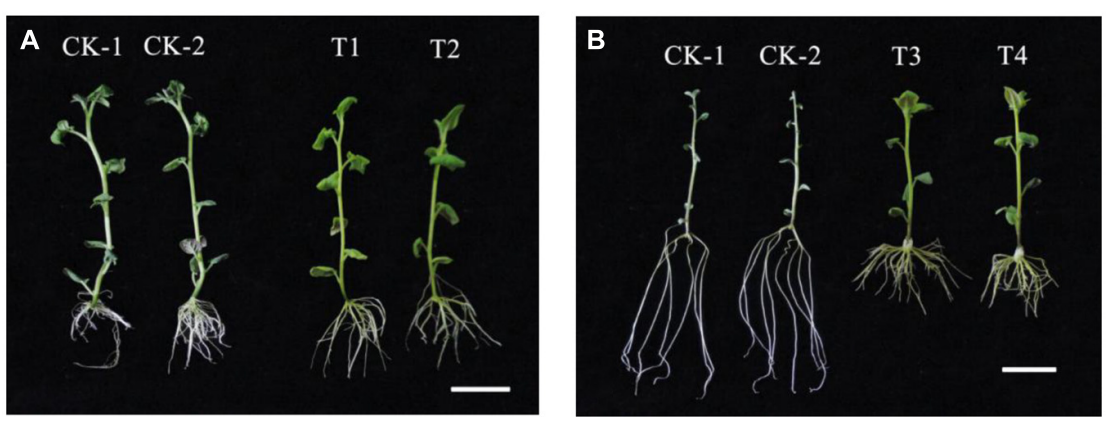

FIGURE 5 | The phenotypic characteristics of the transgenic potato plants. (A) The transgenic potato cultivar "Gannongshu 2." CK: non-transgenic potato as negative control; T1-T2: the transgenic potato plant. (B) The transgenic potato cultivar "Kexin 3." CK: non-transgenic potato as negative control; T3-T4: the transgenic potato plant. The scale bars are of both $2 \mathrm{~cm}$.
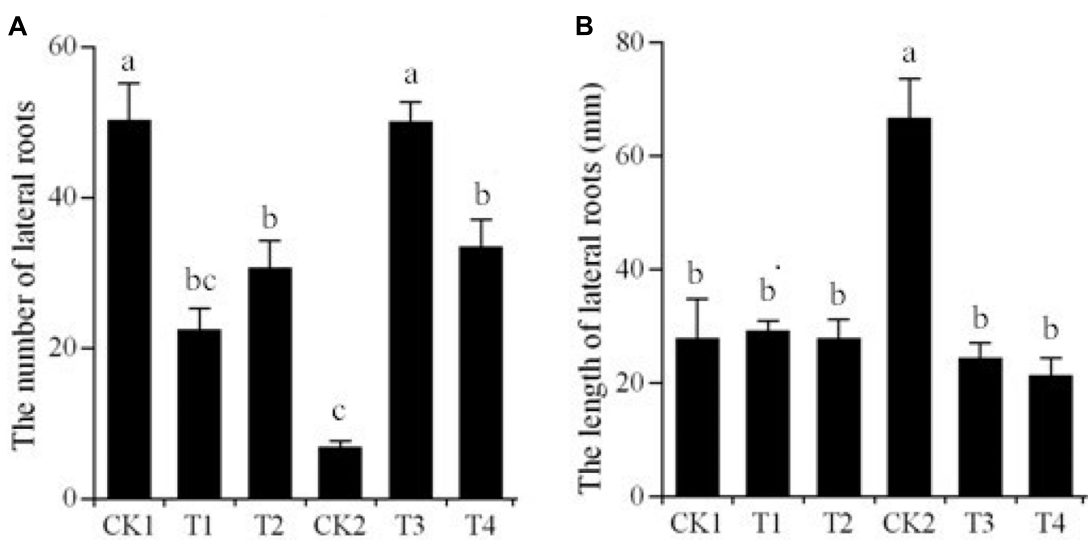

FIGURE 6 | Changes of lateral root number and length of the transgenic potato plants. (A) The number of lateral roots. CK1: non-transgenic potato "Gannongshu 2" as negative control; T1-T2: the transgenic potato transformed with the vector pCPB121-miR164; CK2: non-transgenic potato "Kexin 3" as negative control; T3-T4: the transgenic potato plant transformed with the vector pCPB-NAC262. (B) The length of lateral roots. CK1: non-transgenic potato "Gannongshu 2" as negative control; T1-T2: the transgenic potato transformed with the vector pCPB121-miR164; CK2: non-transgenic potato "Kexin 3" as negative control; T3-T4: the transgenic potato plant transformed with the vector pCPB-NAC262. Values are shown as mean \pm SD from five biological replicates and different letters above the columns indicate significant differences at $p \leq 0.05$ levels with corresponding CK.

thaliana miR164a and miR164b expressed lower miR164 and higher NAC1 mRNA levels lead to more lateral root occurred, while induced expression in wild type plants expressed miR164 can lead to NAC1 mRNA levels suppressed, and then reduce the occurrence of lateral root (Tang et al., 2012).

Researches (He et al., 2005; Quach et al., 2014; Chen et al., 2016) demonstrated that a number of abiotic stress upregulated NAC proteins can promote growth or alter architecture of root, when they are constitutively overexpressed in the transgenic plants, suggesting NAC gene roles in modulating growth and architecture of root under osmotic stress. For instance, the drought-resistant rice variety had a larger and more highly branched root system than the drought-sensitive variety (Price et al., 1997). We also found that the transgenic potato plants with StNAC262 gene had larger root system with longer and more lateral roots than the WT plants when they were grown under osmotic stress (Figures 5, 6), indicating a possible role for StNAC262 regulating root system under osmotic stress and thereby improving tolerance to osmotic stress. It is reported that the conserved features of miRNA upon osmotic stress in Triticeae (Budak and Akpinar, 2011). The requirement of complementarity for target recognition, contributed to identify the putative targets of miRNAs in plants (Budak and Akpinar, 2015). It is likely that miRNA genes can change their expression under drought conditions, which in turn leads to the change in miRNAs expression and ultimately that of miRNAs' targets (Reyes and Chua, 2007; Trindade et al., 2010). Therefore, in individual plant species, the miRNA targets need to be identified. Target validation can also provide functional evidences for the conserved or specific miRNAs in plant.

The potato cultivar "Gannongshu 2," which naturally produces more lateral roots, and "Kexin 3," which naturally produces fewer lateral roots, were used in the experiments reported here. Overexpression of StNAC262 in the Kexin 3 variety produced an increased number of lateral roots thus completely changing the natural tendency of this variety to now produce less lateral roots. Likewise, overexpression of Stu-mi164 in the "Gannongshu 2 " variety developed a reduced number of lateral roots thus 
completely changing the natural tendency of this variety to now produce less lateral roots. So, it appears that a mechanism to deal with osmotic stress in potato is through the production of extra lateral roots, which is a process controlled by StNAC262 and its modulator Stu-mi164. Plants having a more developed root system possess a greater ability to absorb water, so the osmotic stress plants can absorb more water from the soil to reduce the damage caused by a lack of water. Therefore, it is possible to improve the resistant ability of plants to osmotic stress by changing the lateral root number.

\section{AUTHOR CONTRIBUTIONS}

HS and NZ conceived and designed the experiments. LZ, LY, JY, $\mathrm{XZ}$, and XT performed the laboratory experiments. LZ, LY, NZ,

\section{REFERENCES}

Akpinar, B. A., Kantar, M., and Budak, H. (2015). Root precursors of microRNAs in wild emmer and modern wheats show major differences in response to drought stress. Funct. Integr. Genomics 15, 587-598. doi: 10.1007/s10142-0150453-0

Alliotte, T., Tire, C., Engler, G., Peleman, J., Caplan, A., Van Montagu, M., et al. (1989). An auxin-regulated gene of Arabidopsis thaliana encodes a DNA-binding protein. Plant Physiol. 89, 743-752. doi: 10.1104/pp.89.3.743

Bartel, B., and Bartel, D. P. (2003). MicroRNAs: at the root of plant development. Plant Physiol. 132, 709-717. doi: 10.1104/pp.103.023630

Bhardwaj, A. R., Joshi, G., Pandey, R., Kukreja, B., Goel, S., Jagannath, A., et al. (2014). A genome-wide perspective of miRNAome in response to high temperature, salinity and drought stresses in Brassica juncea (Czern) L. PLoS One 9:e92456. doi: 10.1371/journal.pone.0092456

Bonnet, E., Wuyts, J., Rouze, P., and Van, Y. (2004). Detection of 91 potential conserved plant microRNAs in Arabidopsis thaliana and Oryza sativa identifies important target genes. Proc. Natl. Acad. Sci. U.S.A. 101, 11511-11516. doi: 10.1073/pnas.0404025101

Budak, H., and Akpinar, A. (2011). Dehydration stress-responsive miRNA in Brachypodium distachyon: evident by genome-wide screening of microRNAs expression. OMICS 15, 791-799. doi: 10.1089/omi.2011.0073

Budak, H., and Akpinar, B. A. (2015). Plant miRNAs: biogenesis, organization and origins. Funct. Integr. Genomics 15, 523-531. doi: 10.1007/s10142-015-0451-2

Budak, H., Kantar, M., Bulut, R., and Akpinar, B. A. (2015). Stress responsive miRNAs and isomiRs in cereals. Plant Sci. 235, 1-13. doi: 10.1016/j.plantsci. 2015.02.008

Burcu, A., Peter, L., and Hikmet, B. (2016). Abiotic stress miRNomes in the Triticeae. Funct. Integr. Genomics 17, 145-170. doi: 10.1007/s10142-016-0525-9

Chapman, E. J., Prokhnevsky, A. I., Gopinath, K., Dolja, V. V., and Carrington, J. C. (2004). Viral RNA silencing suppressors inhibit the microRNA pathway at an intermediate step. Gene Dev. 18, 1179-1186. doi: 10.1101/gad.1201204

Chen, X. (2004). A microRNA as a translational repressor of APETALA2 in Arabidopsis flower development. Science 303, 2022-2025. doi: 10.1126/science. 1088060

Chen, X. D., Cheng, J. F., Chen, L., Zhang, G., Huang, H., Zhang, Y., et al. (2016). Auxin-independent NAC pathway acts in response to explant-specific wounding and promotes root tip emergence during de novo root organogenesis in Arabidopsis. Plant Physiol. 170, 2136-2145. doi: 10.1104/pp.15.01733

Dai, X., and Zhao, P. X. (2011). psRNATarget: a plant small RNA target analysis server. Nucleic Acids Res. 39, W155-W159. doi: 10.1093/nar/gkr319

Deng, P., Wang, L., Cui, L., Feng, K., Liu, F., Du, X., et al. (2015). Global identification of microRNAs and their targets in barley under salinity stress. PLoS One 10:e0137990. doi: 10.1371/journal.pone.0137990

Duval, M., Hsieh, T. F., Kim, S. Y., and Thomas, T. L. (2002). Molecular characterization of AtNAM: a member of the Arabidopsis NAC domain superfamily. Plant Mol. Biol. 50, 237-248. doi: 10.1023/A:1016028530943 and $\mathrm{AC}-\mathrm{U}$ performed the data analysis and interpretation. LZ, LY, $\mathrm{AC}-\mathrm{U}$, and $\mathrm{HS}$ wrote the paper.

\section{FUNDING}

This research was supported by the National Natural Science Foundation of China (31460370 and 31660416) and the "Fuxi Talent" Plan of Gansu Agricultural University (FXRC20130102).

\section{SUPPLEMENTARY MATERIAL}

The Supplementary Material for this article can be found online at: https://www.frontiersin.org/articles/10.3389/fpls.2018.00383/ full\#supplementary-material

Fang, Y., Xie, K., and Xiong, L. (2014). Conserved miR164-targeted NAC genes negatively regulate drought resistance in rice. J. Exp. Bot. 65, 2119-2135. doi: $10.1093 /$ jxb/eru072

Ferdous, J., Hussain, S. S., and Shi, B. J. (2015). Role of micro RNAs in plant drought tolerance. Plant Biotechnol. J. 13, 293-305. doi: 10.1111/pbi.12318

Griffiths, S., Grocock, R. J., van Dongen, S., Bateman, A., and Enright, A. J. (2006). miRBase: microRNA sequences, targets and gene nomenclature. Nucleic Acids Res. 34, D140-D144. doi: 10.1093/nar/gkj112

Guo, H. S., Xie, Q., and Fei, J. F. (2005). MicroRNA directs mRNA cleavage of the transcription factor NAC1 to downregulate auxin signals for Arabidopsis lateral root development. Plant Cell 17, 1376-1386. doi: 10.1105/tpc.105.030841

He, X. J., Mu, R. L., Cao, W. H., Zhang, Z. G., Zhang, J. S., and Chen, S. Y. (2005). AtNAC2, a transcription factor downstream of ethylene and auxin signaling pathways, is involved in salt stress response and lateral root development. Plant 44, 903-916. doi: 10.1111/j.1365-313X.2005.02575.x

Hikmet, B., Babar, H., Zaeema, K., Neslihan, Z. O., and Naimat, U. (2015). From genetics to functional genomics: improvement in drought signaling and tolerance in wheat. Front. Plant Sci. 6:1012. doi: 10.3389/fpls.2015.01012

Hu, H. H., Dai, M. Q., Yao, J. L., Xiao, B. Z., Li, X. H., Zhang, Q. F., et al. (2006). Overexpressing aNAM, ATAE and CUC (NAC) transcription factor enhances drought resistance and salt tolerance in rice. Proc. Natl. Acad. Sci. U.S.A. 103, 12987-12992. doi: 10.1073/pnas.0604882103

Hu, R., Qi, G., Kong, Y., Kong, D., Gao, Q., and Zhou, G. (2010). Comprehensive analysis of NAC domain transcription factor gene family in Populus trichocarpa. BMC Plant Biol. 10:145. doi: 10.1186/1471-2229-10-145

Hwang, E. W., Shin, S. J., Park, S. C., Jeong, M. J., and Kwon, H. B. (2011). Identification of miR172 family members and their putative targets responding to drought stress in Solanum tuberosum. Genes Genomics 33, 105-110. doi: 10.1007/s13258-010-0135-1

Jin, J. P., Zhang, H., Kong, L., Gao, G., and Luo, J. C. (2014). Plant TFDB 3.0: a portal for the functional and evolutionary study of plant transcription factors. Nucleic Acids Res. 42, D1182-D1187. doi: 10.1093/nar/gkt1016

Jones-rhoades, M. W., and Bartel, D. P. (2004). Computational identification of plant microRNAs and their targets, including a stress-induced miRNA. Mol. Cell 14, 787-799. doi: 10.1016/j.molcel.2004.05.027

Kantar, M. (2010). Regulation of barley miRNAs upon dehydration stress correlated with target gene expression. Funct. Integr. Genomics 10, 493-507. doi: 10.1007/s10142-010-0181-4

Kantar, M., Lucas, S. J., and Budak, H. (2011). miRNA expression patterns of Triticum dicoccoides in response to shock drought stress. Planta 233, 471-484. doi: 10.1007/s00425-010-1309-4

Larue, C. T., Wen, J. Q., and Walker, J. C. (2009). A microRNA-transcription factor module regulates lateral organ size and patterning in Arabidopsis. Plant J. 58, 450-463. doi: 10.1111/j.1365-313X.2009.03796.x

Laufs, P., Peaucelle, A. M., Orrin, H., and Traas, J. (2004). MicroRNA regulation of the CUC genes is required for boundary size control in Arabidopsis meristems. Development 131, 4311-4322. doi: 10.1242/dev.01320 
Liu, H. H., Tian, X., Li, Y. J., Wu, C. A., and Zheng, C. C. (2008). Microarraybased analysis of stress-regulated microRNAs in Arabidopsis thaliana. RNA 14, 836-843. doi: 10.1261/rna.895308

Liu, Q. L., Xu, K. D., Zhao, L. J., Pan, Y. Z., Jiang, B. B., Zhang, H. Q., et al. (2011). Overexpression of a novel chrysanthemum NAC transcription factor gene enhances salt tolerance in tobacco. Biotechnol. Lett. 33, 2073-2082. doi: 10.1007/s10529-011-0659-8

Llave, C., Kasschau, K. D., and Rector, M. A. (2002). Endogenous and silencingassociated small RNAs in plants. Plant Cell 14, 1605-1619. doi: 10.1105/tpc. 003210

Mochida, K., Yoshida, T., Sakurai, T., Yamaguchi-Shinozaki, K., Shinozaki, K., and Tran, L. S. P. (2009). In silico analysis of transcription factor repertoire and prediction of stress responsive transcription factors in soybean. DNA Res. 16, 353-369. doi: 10.1093/dnares/dsp023

Nuruzzaman, M., Manimekalai, R., Sharoni, A. M., Satoh, K., Kondoh, H., Ooka, H., et al. (2010). Genome-wide analysis of NAC transcription factor family in rice. Gene 465, 30-44. doi: 10.1016/j.gene.2010.06.008

Ohnishi, T., Sugahara, S., Yamada, T., Kikuchi, K., Yoshiba, Y., Hirano, H. Y., et al. (2005). OsNAC6, a member of the NAC gene family, is induced by various stresses in rice. Genes Genet. Syst. 80, 135-139. doi: 10.1266/ggs. 80.135

Olsen, A. N., Ernst, H. A., Leggio, L. L., and Skriver, K. (2005). NAC transcription factors: structurally distinct, functionally diverse. Trends Plant Sci. 10, 79-87. doi: 10.1016/j.tplants.2004.12.010

Peaucelle, A., Morin, H., Traas, J., and Laufs, P. (2007). Plants expressing a miR164resistant CUC2 gene reveal the importance of post-meristematic maintenance of phyllotaxy in Arabidopsis. Development 134, 1045-1050. doi: 10.1242/dev. 02774

Price, A. H., Tomos, A. D., and Virk, D. S. (1997). Genetic dissection of root growth in rice (Oryza sativa L.) I: a hydrophonic screen. Theor. Appl. Genet. 95, 132-142. doi: 10.1007/s001220050541

Quach, T. N., Tran, L. S., Vallliyodan, B., and Nguyen, H. T. (2014). Functional analysis of water stress-responsive soybean GmNAC003 and GmNAC004 transcription factors in lateral root development in Arabidopsis. PLoS One 9:e84886. doi: 10.1371/journal.pone.0084886

Reyes, J. L., and Chua, N. H. (2007). ABA induction of mi R159 controls transcript levels of two MYB factors during Arabidopsis seed germination. Plant J. 49, 592-606. doi: 10.1111/j.1365-313X.2006.02980.x

Rhoades, M. W., Reinhart, B. J., and Lim, L. P. (2002). Prediction of plant microRNA targets. Cell 110, 513-520. doi: 10.1016/S0092-8674(02)00863-2

Rushton, P. J., Bokowiec, M. T., Han, S., Zhang, H., Brannock, J. F., Chen, X., et al. (2008). Tobacco transcription factors: novel insights into transcriptional regulation in the Solanaceae. Plant Physiol. 147, 280-295. doi: 10.1104/pp.107. 114041

Saini, S., Dongen, H. K., and Enrigh, S. V. (2008). miRBase: tools for microRNA genomics. Nucleic Acids Res. 36, D154-D158.

Shinozaki, K., and Yamaguchi, K. (2007). Gene networks involved in drought stress response and tolerance. J. Exp. Bot. 58, 221-227. doi: 10.1093/jxb/erl164

Si, H. J., Xie, C. H., and Liu, J. (2003). An efficient protocol for Agrobacteriummediated transformation of microtuber and the introduction of antisense class I patatin gene into potato. Acta Agron. Sin. 29, 801-805.

Souer, E., van Houwelingen, A., Kloos, D., Mol, J., and Koes, R. (1996). The no apical meristem gene of petunia is required for pattern formation in embryos and flowers and is expressed at meristem and primordia boundaries. Cell 85 , 159-170. doi: 10.1016/S0092-8674(00)81093-4

Sunkar, R., and Zhu, J. K. (2004). Novel and stress-regulated microRNAs and other small RNAs from Arabidopsis. Plant Cell 16, 2001-2019. doi: 10.1105/tpc.104. 022830

Tang, Y., Liu, M., Gao, S., Zhang, Z., Zhao, X., Zhao, C., et al. (2012). Molecular characterization of novel TaNAC genes in wheat and overexpression of
TaNAC2a confers drought tolerance in tobacco. Physiol. Plant. 144, 210-224. doi: 10.1111/j.1399-3054.2011.01539.x

Thao, N. P., and Tran, L. S. (2012). Potentials toward genetic engineering of drought-tolerant soybean. Crit. Rev. Biotechnol. 32, 349-362. doi: 10.3109/ 07388551.2011 .643463

Tran, L., Nakashima, K., Sakuma, Y., Simpson, S. D., Fujita, Y., Maruyama, K., et al. (2004). Isolation and functional analysis of Arabidopsis stress-inducible NAC transcription factors that bind to a drought responsive cis-element in the early responsive to dehydration stress promoter. Plant Cell 6, 2481-2498. doi: 10.1105/tpc.104.022699

Trindade, I., Capitao, C., Dalmay, T., Fevereiro, M. P., and Santos, D. M. (2010). miR398 and mi R408 are up-regulated in response to water deficit in Medicago truncatula. Planta 231, 705-716. doi: 10.1007/s00425-009-1078-0

Voinnet, O. (2009). Origin, biogenesis, and activity of plant microRNAs. Cell 136, 669-687. doi: 10.1016/j.cell.2009.01.046

Wang, X. Q., Shen, X., He, Y. M., Ren, T. N., Wu, W. T., and Xi, T. (2011). An optimized freeze-thaw method for transformation of Agrobacterium tumefaciens EHA 105 and LBA4404. Pharm. Biotechnol. 18, 382-386.

Wei, L., Zhang, D., Xiang, F., and Zhang, Z. (2009). Differentially expressed miRNAs potentially involved in the regulation of defense mechanism to drought stress in maize seedlings. Int. J. Plant Sci. 170, 979-989. doi: 10.1086/ 605122

Xie, Q., Frugis, G., Colgan, D., and Chua, N. H. (2000). Arabidopsis NAC1 transduces auxin signal downstream of TIR1 to promote lateral root development. Genes Dev. 14, 3024-3036. doi: 10.1101/gad.852200

Yamaguchi-Shinozaki, K., and Shinozaki, K. (2006). Transcriptional regulatory networks in cellular responses and tolerance to dehydration and cold stresses. Annu. Rev. Plant Biol. 57, 781-803. doi: 10.1146/annurev.arplant.57.032905. 105444

Yang, S., Vanderbeld, B., Wan, J., and Huang, Y. (2010). Narrowing down the targets: towards successful genetic engineering of drought-tolerant crops. Mol. Plant 3, 469-490. doi: 10.1093/mp/ssq016

Yoo, S. Y., Kim, Y., Kim, S. Y., Lee, J. S., and Ahn, J. H. (2007). Control of flowering time and cold response by a NAC-domain protein in Arabidopsis. PLoS One 2:e642. doi: 10.1371/journal.pone.0000642

Zhang, N., Si, H. J., and Wang, D. (2005). Cloning of rd29A gene promoter from Arabidopsis thaliana and its application in stress-resistance transgenic potato. Acta Agron. Sin. 31, 159-164.

Zhang, N., Yang, J., Wang, Z., Wen, Y., Wang, J., He, W., et al. (2014). Identification of novel and conserved microRNAs related to drought stress in potato by deep sequencing. PLoS One 9:e95489. doi: 10.1371/journal.pone.0095489

Zhong, R., Richardson, E. A., and Ye, Z. H. (2007). Two NAC domain transcription factors, SND1 and NST1, function redundantly in regulation of secondary wall synthesis in fibers of Arabidopsis. Planta 225, 1603-1611. doi: 10.1007/s00425007-0498-y

Zhou, L., Liu, Y., Liu, Z., Kong, D., Duan, M., and Luo, L. (2010). Genome-wide identification and analysis of drought-responsive microRNAs in Oryza sativa. J. Exp. Bot. 61, 4157-4168. doi: 10.1093/jxb/erq237

Conflict of Interest Statement: The authors declare that the research was conducted in the absence of any commercial or financial relationships that could be construed as a potential conflict of interest.

Copyright (c) 2018 Zhang, Yao, Zhang, Yang, Zhu, Tang, Calderón-Urrea and Si. This is an open-access article distributed under the terms of the Creative Commons Attribution License (CC BY). The use, distribution or reproduction in other forums is permitted, provided the original author(s) and the copyright owner are credited and that the original publication in this journal is cited, in accordance with accepted academic practice. No use, distribution or reproduction is permitted which does not comply with these terms. 